\title{
JSJ decompositions of quadratic Baumslag-Solitar groups
}

\author{
JuAN ALONSO
}

\begin{abstract}
Generalized Baumslag-Solitar groups are defined as fundamental groups of graphs of groups with infinite cyclic vertex and edge groups. Forester [6] proved that in most cases the defining graphs are cyclic JSJ decompositions, in the sense of Rips and Sela. Here we extend Forester's results to graphs of groups with vertex groups that can be either infinite cyclic or quadratically hanging surface groups.
\end{abstract}

20E06, 20E08, 20F65; 57M60

\section{Introduction}

To understand a group $G$, it is often useful to decompose it as an amalgamated free product or an HNN extension over a subgroup that belongs to a well understood class of groups, such as trivial groups, finite groups or cyclic groups. More generally, consider all possible factorizations of $G$ as a graph of groups with edge stabilizers in some single class of groups.

It is often possible to show the existence of a single graph of groups decomposition, from which all of these factorizations can be obtained. This is called a JSJ decomposition of $G$ (over subgroups in the given class), although the notion is imprecise on how the other factorizations of $G$ are to be obtained from the JSJ decomposition. An example is Grushko's theorem, which gives all the maximal decompositions of a finitely generated group over the class of trivial groups (ie the free factorizations).

The letters JSJ stand for Jaco, Shalen and Johannson. Their results in [11;12] can be interpreted as the existence of a JSJ decomposition for 3-manifold groups over subgroups isomorphic to $\mathbb{Z} \times \mathbb{Z}$. It was these works that motivated the study of JSJ decompositions over nontrivial subgroups (ie aside from the Grushko decomposition). Various existence theorems were obtained by Kropholler [13], Rips and Sela [16; 14], Bowditch [2], Dunwoody and Sageev [3], Fujiwara and Papasoglu [7], Dunwoody and Swenson [4] and Scott and Swarup [15]. In [10; 8] Guirardel and Levitt propose a precise definition of JSJ decomposition, which is verified by the graphs of groups constructed in most of the mentioned works. The constructions in [4] and [15] constitute other 
notions of JSJ decomposition, as does the compatibility JSJ decomposition introduced by Guirardel and Levitt [9].

In this paper we will focus on the JSJ decomposition due to Rips and Sela [14] for finitely presented one-ended groups with infinite cyclic edge stabilizers (stated here as Theorem 2.14).

It is not always clear how to recognize whether a given graph of groups is a JSJ decomposition of its fundamental group. In [6], Forester studied the Generalized Baumslag-Solitar (GBS) groups, which are defined by graphs of groups whose vertex and edge stabilizers are infinite cyclic. He showed that the defining graph of a GBS group is a JSJ decomposition, under mild hypotheses.

In this paper we introduce the quadratic Baumslag-Solitar (QBS) groups. They are defined by graphs of groups whose edge groups are infinite cyclic, and whose vertex groups can be either infinite cyclic or quadratically hanging (see Definition 2.7) surface groups. It is clear that the GBS groups are a subclass of the QBS groups. We extend Forester's result to the class of QBS groups, ie we show that the defining graph of a QBS group is a JSJ decomposition, under some conditions. Specifically, the main theorem in this paper is the following.

Theorem 1.1 Let $\Gamma$ be a $Q B S$ graph, $G=\pi_{1}(\Gamma)$. Suppose that $\Gamma$ is reduced, has no leaves, and satisfies the following conditions:

(1) Each edge $e$ of $\Gamma$ has labels $m_{e}^{+}, m_{e}^{-}>1$.

(2) Each GBS component $\Gamma_{i}$ of $\Gamma$ is reduced, and $T_{\Gamma_{i}}$ is not a point or a line.

Then $\Gamma$ is a Rips-Sela JSJ decomposition for $G$.

A $Q B S$ graph is the defining graph of a QBS group. See Section 2.2 for the definition of a reduced graph of groups. For the edge labels, see Definition 5.1. The GBS components and the leaves of a QBS graph are defined at the beginning of Section 6.

The paper is organized as follows: In Section 2 we review the basics of the Rips-Sela JSJ decomposition. There we discuss universality and unfoldedness, the two main conditions for a graph of groups (with cyclic edge groups) to be a JSJ decomposition (in the sense of Rips and Sela). In Section 3 we recall the results of Forester about GBS groups, which we will need when dealing with QBS groups. In Section 4 we prove a general criterion for unfoldedness, Theorem 4.2, in the same fashion of the one by Forester [6, Proposition 2.17]. Section 5 is devoted to Theorem 5.5. This theorem applies to general graphs of groups with cyclic edge stabilizers, and it allows us to 
show the universality of the whole graph from the universality of certain subgraphs. Finally, in Section 6 we introduce the QBS groups, and prove they are one-ended with the exception of $\mathbb{Z}$ (see Proposition 6.1). We also give the proof of Theorem 1.1. In this proof we combine Theorem 5.5 with the results of Forester (outlined in Section 3) to show universality, and we use Theorem 4.2 to prove unfoldedness.

\section{Review of the JSJ decomposition}

\subsection{Graphs of groups and Bass-Serre theory}

Bass-Serre theory is the most fundamental prerequisite for understanding the JSJ decomposition. This is a very quick review, a comprehensive treatment is given in the book by Serre [17].

For a graph we understand a pair of sets $\Gamma=(V, E)$, the vertex and edge set of $\Gamma$ respectively, together with two maps $s, t: E \rightarrow V$, which give the source and target of an edge. If $e \in E$ is such an edge, the vertices $s(e), t(e)$ will be called the endpoints of $e$. Thus our graphs have oriented edges, and admit loops (edges $e$ with $s(e)=t(e)$ ) and multiple edges (different edges having the same endpoints). We will usually drop the maps $s$ and $t$ from the notation, thus we will say an edge $e$ has endpoints $v^{+}, v^{-}$ to mean that $s(e)=v^{-}$and $t(e)=v^{+}$.

Definition 2.1 A graph of groups consists of the following:

(1) a connected graph $\Gamma$;

(2) a group $G_{v}$ for each vertex $v$ of $\Gamma$;

(3) a group $G_{e}$ for each edge $e$ of $\Gamma$, and two injective homomorphisms

$$
\begin{gathered}
\partial_{e}^{+}: G_{e} \rightarrow G_{v^{+}} \\
\partial_{e}^{-}: G_{e} \rightarrow G_{v^{-}}
\end{gathered}
$$

where $v^{+}, v^{-}$are the endpoints of $e$.

This is denoted by $\left(\Gamma, G, \partial^{+}, \partial^{-}\right)$, or simply by $\Gamma$. Note that even if the endpoints of an edge $e$ agree, ie $v^{+}=v^{-}$, there are two different maps $\partial_{e}^{+}$and $\partial_{e}^{-}$, one for the source and one for the target of $e$. 
If $T$ is a spanning tree for $\Gamma$, let $\pi_{1}(\Gamma, T)$ be defined by the following presentation.

- Generators: the elements of $G_{v}$ for the vertices $v \in V(\Gamma)$, and an element $t_{e}$ for each edge $e \in E(\Gamma), e \notin T$.

- Relations: the relations in $G_{v}$ for each vertex $v$, and

$$
\begin{aligned}
\partial_{e}^{+}(g) & =\partial_{e}^{-}(g) \quad \text { for } e \in T, g \in G_{e}, \\
t_{e} \partial_{e}^{+}(g) t_{e}^{-1} & =\partial_{e}^{-}(g) \quad \text { for } e \in E(\Gamma), e \notin T, g \in G_{e} .
\end{aligned}
$$

This group is called the fundamental group of $\Gamma$. It does not depend on the spanning tree $T$.

Proposition 2.2 If $T, S$ are two spanning trees for $\Gamma$, then $\pi_{1}(\Gamma, T) \cong \pi_{1}(\Gamma, S)$.

Thus we often drop $T$ from the notation. When $G$ is a group and $G \cong \pi_{1}(\Gamma)$ for a graph of groups $\Gamma$, we say that $\Gamma$ is a splitting of $G$. Note that one-edge splittings correspond to decompositions of $G$ as an amalgamated product or an HNN extension. If $\Gamma$ is a graph of groups and $A \subset \Gamma$ is a connected subgraph, let $\Gamma^{*}=\Gamma / A$ be the graph obtained by collapsing $A$ to a vertex, which we call $w$. Put $G_{w}=\pi_{1}(A)$ and leave the same groups in the noncollapsed vertices and edges. This defines a graph of groups in $\Gamma^{*}$.

Proposition 2.3 If $A \subset \Gamma$ is a connected subgraph and $\Gamma^{*}=\Gamma / A$ as above, then $\pi_{1}(\Gamma) \cong \pi_{1}\left(\Gamma^{*}\right)$.

We say that $\Gamma$ is a refinement of the splitting $\Gamma^{*}$. Through this proposition, we can see general splittings as iteration of amalgamated products and HNN extensions.

The following is the main result in Bass-Serre theory. It relates the splittings of a group with its actions on simplicial trees, and it is crucial for both the theory of JSJ decompositions and the results on this paper.

Theorem 2.4 (Bass-Serre) Given a group $G$, there is a correspondence between the splittings of $G$ as a graph of groups and the actions of $G$ on simplicial trees without edge inversions. If $G \curvearrowright X$ is such an action, then a corresponding splitting can be constructed as follows:

- $\quad$ The underlying graph is $\Gamma=X / G$.

- If $\tilde{x} \in X$ is a vertex or edge, and $x \in \Gamma$ is its projection, then $G_{x}$ is isomorphic to $\operatorname{Stab}_{G}(\tilde{x})$. 
Since the action on $X$ is without edge inversions, the edges of $\Gamma=X / G$ can be given an orientation. We omitted the edge maps in the statement, but they are straightforward. Namely, if we identify $G_{x}$ with $\operatorname{Stab}_{G}(\tilde{x})$ for some specific $\tilde{x}$, these maps are subgroup inclusions possibly composed with conjugations. The action corresponding to a splitting $\Gamma$ under this theorem will be denoted by $T_{\Gamma}$, the Bass-Serre tree of $\Gamma$.

Given a simplicial $G$-tree $X$, a subgroup $H \leq G$ acts elliptically on $X$, or is an elliptic subgroup with respect to $X$, if there is a point in $X$ that is fixed by every element of $H$. Note that if $X=T_{\Gamma}$ is the Bass-Serre tree of a graph of groups $\Gamma$, then a subgroup of $G=\pi_{1}(\Gamma)$ acts elliptically on $X$ if and only if it is conjugate into one of the vertex groups of $\Gamma$.

Let $X, Y$ be simplicial $G$-trees. A morphism $f: X \rightarrow Y$ is a $G$-equivariant map, which can be made simplicial by subdividing the edges of $X$. The following fact is widely known, and not hard to prove.

Proposition 2.5 There is a morphism $X \rightarrow Y$ if and only if every elliptic subgroup of $X$ is also elliptic in $Y$.

\subsection{Elementary deformations, foldings}

Here we introduce some important transformations on graphs of groups.

Let $\Gamma$ be a graph of groups. Let $e$ be an edge of $\Gamma$ and $v^{+}, v^{-}$its endpoints. First suppose that $v^{+} \neq v^{-}$and $\partial_{e}^{-}$is an isomorphism. That is, $G_{e}=G_{v^{-}}=C$ and $G_{v^{+}}=A$ with $C \subset A$. In this situation, the collapse of the edge $e$ is called an elementary collapse. Note that $v^{+}$and $v^{-}$are identified to a single vertex $\bar{v}$, and $G_{\bar{v}}=A$ (through the isomorphism $A *_{C} C \cong A$ ).

The inverse of an elementary collapse is called an elementary expansion, and these transformations are the elementary deformations, which were introduced by Forester [5].

Again, let $e$ be an edge with different endpoints. Suppose $G_{e}=C \subset C_{1} \subset A=G_{v^{+}}$ and $B=G_{v^{-}}$. Get $\Gamma_{1}$ from $\Gamma$ by redefining $G_{e}=C_{1}$ and $G_{v^{-}}=C_{1} *_{C} B$. We have $\pi_{1}(\Gamma)=\pi_{1}\left(\Gamma_{1}\right)$ by the isomorphism $A *_{C} B \cong A *_{C_{1}}\left(C_{1} *_{C} B\right)$. In this case we say that $\Gamma_{1}$ is a folding of $\Gamma$, and that the folding occurs at the vertex $v^{+}$.

There is another case of folding when $e$ is a loop, ie $v^{+}=v^{-}=v$. Let $G_{e}=C, G_{v}=A$, and suppose that $\partial_{e}^{+}(C) \subset C_{1} \subset A$. This time make $\Gamma_{1}$ with $G_{v}=A *_{C} t_{e} C_{1} t_{e}^{-1}$ and $G_{e}=C_{1}$. The fundamental group is again preserved, and this transformation is also called folding. Making some abuse of notation, we say that the folding occurs at $v^{+}$in the case just described, and at $v^{-}$if we use $\partial_{e}^{-}$instead. 
Looking at the Bass-Serre trees, when there is a folding we have a map $T_{\Gamma} \rightarrow T_{\Gamma_{1}}$, simplicial and equivariant. If $x \in T_{\Gamma}$ is a lift of $v^{+}$with stabilizer $g \mathrm{Ag}^{-1}$, then this map identifies the edges coming from $x$ and projecting to $e$, by the action of $g C_{1} g^{-1}$. Locally at $x$ it looks like "folding". In [1], Bestvina and Feighn explain this from the viewpoint of graphs of groups.

If $e$ is an edge of $\Gamma$, let $\Gamma_{e}$ be the graph of groups obtained by collapsing the components of $\Gamma-e$.

Definition 2.6 A splitting $\Gamma$ is unfolded when one of the following holds:

(1) $\Gamma$ has only one edge, and there is no folding onto it. That is, there is no $\Gamma_{0}$ such that $\Gamma$ is obtained as a folding of $\Gamma_{0}$.

(2) $\Gamma$ has several edges, and $\Gamma_{e}$ is unfolded for all of them.

\section{$2.3 \mathbb{Z}$-Splittings, quadratically hanging subgroups}

A $\mathbb{Z}$-splitting of the group $G$ is a splitting whose edge groups are infinite cyclic. That is, a graph of groups $\Gamma$, with $\pi_{1}(\Gamma) \cong G$ and $G_{e} \cong \mathbb{Z}$ for all edges of $\Gamma$.

Definition 2.7 Let $\Gamma$ be a graph of groups. A vertex group $G_{v}$ is quadratically hanging $(Q H)$ if:

(1) $G_{v} \cong \pi_{1}(S)$ where $S$ is a 2 -orbifold, ie it has one of the following presentations: $\left\langle a_{1}, \ldots, a_{g}, b_{1}, \ldots, b_{g}, p_{1}, \ldots, p_{m}, s_{1}, \ldots, s_{n} \mid s_{i}^{k_{i}}=1, \Pi_{k} p_{k} \Pi_{i} s_{i} \Pi_{j}\left[a_{j}, b_{j}\right]=1\right\rangle$, $\left\langle a_{1}, \ldots, a_{g}, p_{1}, \ldots, p_{m}, s_{1}, \ldots, s_{n} \mid s_{i}^{k_{i}}=1, \Pi_{k} p_{k} \Pi_{i} s_{i} \Pi_{j} a_{j}^{2}=1\right\rangle$.

We require $S$ to be different from the disk, the cylinder, and a disk with one cone-point.

(2) The edges from $v$ are in correspondence with the components of $\partial S$. Moreover, if these edges are $e_{1}, \ldots, e_{m}$, then we have $\partial_{e_{i}}: G_{e_{i}} \rightarrow\left\langle p_{i}\right\rangle$ (where $p_{i}$ is the boundary loop corresponding to $\left.e_{i}\right)$, and $G_{e_{i}}$ is nontrivial.

Definition 2.8 Let $G$ be a group. Then $P \subset G$ is a $Q H$ subgroup if there is a $\mathbb{Z}$-splitting $\Gamma_{P}$ of $G$ with $P$ occurring as a $\mathrm{QH}$ vertex group.

Our definition of $\mathrm{QH}$ vertex differs slightly from the one originally used by Rips and Sela in [14], in which they require the maps $\partial_{e_{i}}: G_{e_{i}} \rightarrow\left\langle p_{i}\right\rangle$ to be onto. This does not change the $\mathrm{QH}$ subgroups, since the additional condition on the $\mathrm{QH}$ vertex can be met by performing elementary expansions on $\Gamma_{P}$. 
Definition 2.9 We say that a $\mathbb{Z}$-splitting is reduced if it does not admit elementary collapses, except possibly on the edges connecting to $\mathrm{QH}$ vertices.

Let $\Gamma_{1}, \Gamma_{2}$ be one-edged $\mathbb{Z}$-splittings of $G$, with edge groups $C_{1}, C_{2}$ respectively. That is, $G$ is written as an amalgamation or HNN extension over $C_{i}$. We say that $\Gamma_{1}$ is elliptic in $\Gamma_{2}$ if the subgroup $C_{1}$ acts elliptically in $T_{\Gamma_{2}}$, the Bass-Serre tree of $\Gamma_{2}$. Otherwise, we say that $\Gamma_{1}$ is hyperbolic in $\Gamma_{2}$.

Proposition 2.10 [14, Theorem 2.1] Let $G$ be freely indecomposable, and $\Gamma_{1}, \Gamma_{2}$ be one-edged $\mathbb{Z}$-splittings of $G$. Then $\Gamma_{1}$ is elliptic in $\Gamma_{2}$ if and only if $\Gamma_{2}$ is elliptic in $\Gamma_{1}$.

\subsection{The Rips-Sela JSJ decomposition}

We will now state the fundamental theorem of Rips and Sela, which proves the existence of certain $\mathbb{Z}$-splittings that will be called JSJ decompositions. It applies to one-ended groups, that are defined as follows.

Definition 2.11 A space $X$ is one-ended if there is an increasing sequence of compact sets $K_{n}$, such that $X=\bigcup_{n} K_{n}$ and $X-K_{n}$ is connected for all $n$.

Definition 2.12 A group $G$ is one-ended if one/all of its Cayley graphs is/are oneended. Equivalently, for $G$ finitely generated, if it acts freely and cocompactly on a one-ended space.

Consider a class of groups $\mathcal{A}$, such as trivial, finite or cyclic groups. We say that a group $G$ splits over $\mathcal{A}$ if it admits a nontrivial graph of groups decomposition with edge groups in $\mathcal{A}$. For example, $G$ splits over infinite cyclic groups if it admits a nontrivial $\mathbb{Z}$-splitting.

According to a theorem of Stallings [18], a finitely generated infinite group is one-ended if and only if it does not split over finite groups. Thus it makes sense to study the splittings over infinite cyclic groups, $\mathbb{Z}$-splittings, of such a group as a next step.

Definition 2.13 A simple closed curve in a 2-orbifold $S$ is weakly essential if it is not nullhomotopic, nor boundary parallel, nor the core of a Möbius band embedded in $S$, and does not circle around a branching point. 
Theorem 2.14 (Rips-Sela) Let $G$ be a finitely presented one-ended group. Then there is a reduced, unfolded $\mathbb{Z}$-splitting $\Gamma$ of $G$ satisfying the following conditions:

(1) (a) A vertex group of $\Gamma$ can either be a $Q H$ vertex group, or be elliptic in every $\mathbb{Z}$-splitting of $G$.

(b) Edge groups are elliptic in every $\mathbb{Z}$-splitting of $G$.

(c) Every maximal $Q H$ subgroup of $G$ is conjugate to a $Q H$ vertex group of $\Gamma$.

(2) Let $\Gamma_{1}$ be a one-edged $\mathbb{Z}$-splitting of $G$, with edge group $C$. Suppose that $\Gamma_{1}$ is hyperbolic in some other one-edged $\mathbb{Z}$-splitting. Then there is a $\mathrm{QH}$ vertex group $G_{v}=\pi_{1}(S)$ of $\Gamma$, and a weakly essential simple closed curve $\gamma \subset S$ such that $C$ is conjugate to the group generated by $[\gamma] \in G_{v} \subset G$.

(3) If $\Gamma_{1}$ is a one-edged $\mathbb{Z}$-splitting of $G$ that is elliptic in every other one-edged $\mathbb{Z}$-splitting, then there is a morphism $T_{\Gamma} \rightarrow T_{\Gamma_{1}}$.

(4) Let $\Gamma_{1}$ be any $\mathbb{Z}$-splitting of $G$. Then there is a $\mathbb{Z}$-splitting $\hat{\Gamma}$, which is a refinement of $\Gamma$ obtained by splitting some $Q H$ vertex groups along weakly essential simple closed curves, and a morphism $T_{\widehat{\Gamma}} \rightarrow T_{\Gamma_{1}}$.

A splitting $\Gamma$ as in the theorem is called a cyclic JSJ decomposition, or Rips-Sela JSJ decomposition of $G$. Here we will consider only this version of JSJ decomposition. Due to our definition of QH vertices (Definition 2.7), our JSJ decompositions may differ a bit from the ones in [14], but they agree after elementary expansions at the QH vertices.

Condition (4) in the theorem is called universality. It says how every $\mathbb{Z}$-splitting of a group $G$ can be obtained from a JSJ decomposition. Also, it is because of universality that the splitting in the theorem verifies the general definition of a JSJ decomposition (over infinite cyclic groups), given by Guirardel and Levitt in [10; 8]. Although we will not need that definition here.

There is some redundancy in the conditions for a Rips-Sela JSJ decomposition, as the following proposition shows.

Proposition 2.15 Let $G$ be a one-ended group. Suppose $\Gamma$ is a reduced $\mathbb{Z}$-splitting of $G$ satisfying universality, as in (4) of Theorem 2.14. Then it also satisfies conditions (1), (2) and (3) of Theorem 2.14.

Proof For (a) and (b) of condition (1), let $\Gamma_{1}$ be any $\mathbb{Z}$-splitting of $G$. Let $\hat{\Gamma}$ and $f: T_{\widehat{\Gamma}} \rightarrow T_{\Gamma_{1}}$ be the refinement and the morphism given by universality. If $G_{v}$ is a vertex group of $\Gamma$ that is not $\mathrm{QH}$, then it is still elliptic in $\widehat{\Gamma}$, and so it is elliptic in $\Gamma_{1}$. 
This proves (a). The edge groups of $\Gamma$ are also elliptic in $\hat{\Gamma}$, and so they are elliptic in $\Gamma_{1}$. This gives (b).

Now we prove (2). Let $\Gamma_{1}$ be a one-edged $\mathbb{Z}$-splitting of $G$ that is hyperbolic in some other $\mathbb{Z}$-splitting. Let $\hat{\Gamma}$ be the refinement of $\Gamma$ given by condition (4), and $f: T_{\widehat{\Gamma}} \rightarrow T_{\Gamma_{1}}$ the corresponding morphism. Take $e$ an edge in $T_{\Gamma_{1}}$, let $C=\operatorname{Stab}_{G}(e)$ be its stabilizer subgroup and $K=f^{-1}(e)$ be its preimage under $f$. There are two kinds of edges in $\hat{\Gamma}$ : those that were already present in $\Gamma$, and those that were obtained by cutting the surfaces of $\mathrm{QH}$ vertices along simple closed curves. Since $f(K)=e, K$ is not a single point and it meets the interior of an edge $e_{1}$. Then $\operatorname{Stab}_{G}\left(e_{1}\right) \subset C$. Moreover, since $C$ is cyclic, the generator of $\operatorname{Stab}_{G}\left(e_{1}\right)$ is a power of the one of $C$. If $e_{1}$ was of the first kind, then $C$ would be elliptic in every $\mathbb{Z}$-splitting of $G$, which is a contradiction against our assumption on $\Gamma_{1}$. Thus $e_{1}$ is of the second kind, and $K$ does not meet the interior of any edges of the first kind. Let $K^{+}$be the union of the edges $e^{\prime}$ of $T_{\widehat{\Gamma}}$ so that $\operatorname{Stab}_{G}\left(e^{\prime}\right)$ intersects $C$ in a nontrivial subgroup. Then $K^{+}$ is connected and contains $K$. (If $C=\langle c\rangle$, then $K^{+}=\bigcup_{n \geq 1}$ Fix $\left(c^{n}\right)$, which is an increasing union of connected sets). The same reasoning used for $e_{1}$ shows that $K^{+}$ does not contain edges of the first kind. (Recall that an element $g$ is elliptic if and only if $g^{n}$ is elliptic for any $n \neq 0$ ).

Now let $v$ be the QH vertex of $\Gamma$ that corresponds to $e_{1}$. Let $\Gamma_{0}$ be the splitting of $G_{v}=\pi_{1}(S)$ obtained by cutting $S$ along the same simple closed curves as in $\hat{\Gamma}$. Then there is a copy of $T_{\Gamma_{0}}$ embedded in $T_{\widehat{\Gamma}}$ containing $e_{1}$. Notice if $g: T_{\widehat{\Gamma}} \rightarrow T_{\Gamma}$ is the map that collapses all edges of the second kind, then $g$ collapses $T_{\Gamma_{0}}$ to a vertex $w$ in the orbit of $v$. So $\operatorname{Stab}_{G}\left(T_{\Gamma_{0}}\right)=\operatorname{Stab}_{G}(w)$ and it is conjugate to $G_{v}=\pi_{1}(S)$. Observe $K^{+}$must be contained in $T_{\Gamma_{0}}$, since it cannot cross edges of the first kind. In particular, any fixed point of $C$ lies in $T_{\Gamma_{0}}$, and so it is mapped to $w$ by $g$. Thus $C \subset \operatorname{Stab}_{G}(w)$ and is conjugate to $G_{v}$. And $C=\operatorname{Stab}_{G}\left(e_{1}\right)$, since a simple closed curve represents a primitive element of $\pi_{1}(S)$. This proves (2).

Now we will prove (3). The setup is the same as in the previous case, but this time $\Gamma_{1}$ is elliptic in every $\mathbb{Z}$-splitting of $G$. This time, $K$ cannot meet the interior of any edge of the second kind. To see that, suppose $K$ intersects the interior of an edge $e_{1}$ of the second kind. Let $G_{v}=\pi_{1}(S)$ be the $\mathrm{QH}$ vertex group of $\Gamma$ corresponding to $e_{1}$, and let $\alpha$ be the simple closed curve in $S$ such that $\operatorname{Stab}_{G}\left(e_{1}\right)$ conjugates to $\langle[\alpha]\rangle$. Since $K$ meets the interior of $e_{1}$, we have $\operatorname{Stab}_{G}\left(e_{1}\right) \subset C$. Thus, if we write $C=\langle c\rangle$, we get $c^{n}$ is conjugate to $[\alpha]$ for some $n$. Let $\beta$ be a simple closed curve in $S$ that intersects $\alpha$ nontrivially and minimally. Then consider the one-edged splitting $\Gamma_{2}$ of $G$ obtained from $[\beta]$. Since $[\alpha]$ acts hyperbolically on $T_{\Gamma_{2}}$, so does $c$. Thus $\Gamma_{1}$ is hyperbolic in $\Gamma_{2}$ (and viceversa, by Proposition 2.10), which goes against our assumption. So $K$ does not intersect any edges of the second kind. This was shown for $K=f^{-1}(e)$ 
where $e$ was any edge in $T_{\Gamma_{1}}$, so all the edges of the second kind are collapsed to points under $f$. Let $g: T_{\widehat{\Gamma}} \rightarrow T_{\Gamma}$ be the map obtained by collapsing the edges of the second kind. Then $f$ factors through $g$, and so we obtain the morphism in (3).

Finally, for (a), let $H$ be a QH subgroup of $G$. Let $\Gamma_{1}$ be a $\mathbb{Z}$-splitting realizing it as a QH vertex. Write $H=\pi_{1}(S)$ as given by $\Gamma_{1}$. Again, condition (4) gives a morphism $f: T_{\widehat{\Gamma}} \rightarrow T_{\Gamma_{1}}$ for some refinement $\widehat{\Gamma}$ of $\Gamma$ as before.

If $c$ is the class of a boundary component of $S$, then $c$ acts elliptically on $T_{\widehat{\Gamma}}$. To see that, note that some power of $c$ fixes an edge $e$ of $T_{\Gamma_{1}}$ (the incident edge at $v$ corresponding to this boundary curve), and $f^{-1}(e)$ meets the interior of some edge $e_{1}$. We obtain that $\operatorname{Stab}_{G}\left(e_{1}\right) \subset \operatorname{Stab}_{G}(e) \subset\langle c\rangle$, thus $e_{1}$ is fixed by a power of $c$.

Consider the action of $H$ on $T_{\hat{\Gamma}}$ by restriction, and let $\hat{T}$ be a minimal subtree for this action. Then we have the boundary classes of $S$ are elliptic in $\hat{T}$, since they are elliptic in $T_{\widehat{\Gamma}}$.

Consider the decomposition $\Gamma_{H}$ of $H$ induced by $\widehat{T}$. If $e$ is an edge in $\widehat{T}$, then we have $\operatorname{Stab}_{H}(e) \subset \operatorname{Stab}_{G}(e)$, so the edge groups of $\Gamma_{H}$ are either trivial or infinite cyclic. Since the boundary classes of $S$ are elliptic in $\widehat{T}$, then $\Gamma_{H}$ can be extended to $\Gamma_{2}$, a splitting of $G$ obtained by refining $\Gamma_{1}$. And since $G$ is one-ended, all edge groups of $\Gamma_{2}$ are infinite cyclic. Hence all edge groups of $\Gamma_{H}$ are infinite cyclic.

Using Corollary 5.3, $\Gamma_{H}$ is obtained by splitting $S$ along some disjoint, weakly essential simple closed curves. Now, if $e$ is an edge in $\widehat{T}$, then $\operatorname{Stab}_{H}(e)$ is generated

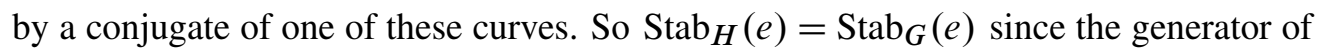
$\operatorname{Stab}_{H}(e)$ is primitive. And it is also hyperbolic in some $\mathbb{Z}$-splitting of $G$, so $e$ is of the second kind.

We conclude as in the proof of (2), obtaining that $H$ is conjugate into $G_{v}$, for $v$ a $\mathrm{QH}$ vertex of $\Gamma$.

Corollary 2.16 Let $G$ be a one-ended group. If $\Gamma$ is a reduced, unfolded $\mathbb{Z}$-splitting of $G$ that verifies universality, as in Theorem 2.14(4), then it is a Rips-Sela JSJ decomposition for $G$.

\section{Generalized Baumslag-Solitar groups}

Here we discuss the results in Forester's paper [6] that are relevant to this paper.

Definition 3.1 A Generalized Baumslag-Solitar $(G B S)$ graph is a graph of groups in which all vertex and edge groups are infinite cyclic. 
Note this is a special case of $\mathbb{Z}$-splitting. A GBS group is a group obtained as a fundamental group of a GBS graph, and a GBS tree is the associated Bass-Serre tree.

Lemma 3.2 [6, Lemma 2.6] Let $\Gamma$ be a GBS graph, $G=\pi_{1}(\Gamma)$. Assume $G \not \mathbb{Z}$, and let $T=T_{\Gamma}$ be the Bass-Serre tree of $\Gamma$. Then:

(1) $G$ is not free;

(2) $G$ acts freely on $T \times \mathbb{R}$;

(3) $G$ is torsion-free, one-ended and has cohomological dimension 2;

(4) $T$ contains an invariant line if and only if $G \cong \mathbb{Z} \rtimes \mathbb{Z}$ (ie either $\mathbb{Z}^{2}$ or the Klein bottle group).

The following is the most general statement about JSJ decompositions of GBS groups.

Theorem 3.3 [6, Theorem 2.15] Let $\Gamma$ be a $G B S$ graph, $G=\pi_{1}(\Gamma)$. Suppose $\Gamma$ is reduced, unfolded, and $T_{\Gamma}$ is not a point or a line $(G \not \mathbb{Z}, \mathbb{Z} \rtimes \mathbb{Z})$. Then $\Gamma$ is a JSJ decomposition of $G$.

In general, it is hard to check wether a splitting is unfolded or not. The following result proves unfoldedness for most GBS graphs.

Proposition 3.4 [6, Proposition 2.17] Let $\Gamma$ be a GBS graph. If every edge group is a proper subgroup of its neighboring vertex groups, then $\Gamma$ is unfolded.

The combination the two last statements permits us to recognize most GBS graphs as JSJ decompositions of their fundamental groups.

\section{Criterion for unfoldedness}

Here we give a criterion for the unfoldedness of a general $\mathbb{Z}$-splitting. It is a generalization of Proposition 3.4, due to Forester, and the proof follows the same lines.

Lemma 4.1 Let $G$ be a freely indecomposable group. Suppose that $\Gamma$ is a $\mathbb{Z}$-splitting of $G, e$ is an edge of $T_{\Gamma}$ with endpoints $v_{0}, v_{1}$ and $H \leq \operatorname{Stab}_{G}\left(v_{1}\right)$ contains $\operatorname{Stab}_{G}(e)$ properly. If $\Gamma_{1}$ is a nontrivial unfolding of $\Gamma_{e}$ at the endpoint $v_{0}$ of $e$, then $H$ cannot be elliptic in $\Gamma_{1}$.

In the statement of the lemma, we abused notation and still called $e, v_{0}$ and $v_{1}$ their respective projections in $\Gamma$ and $\Gamma_{e}$. Recall that $\Gamma_{e}$ is the graph obtained from $\Gamma$ by collapsing all edges but the projection of $e$. 
Proof Let $X$ be the Bass-Serre tree corresponding to $\Gamma_{e}$ and $Y$ the one corresponding to $\Gamma_{1}$. Notice $X$ can be obtained from $T_{\Gamma}$ by collapsing the components of $T_{\Gamma}-G e$. Let $q: T_{\Gamma} \rightarrow X$ be the quotient map, and $f: Y \rightarrow X$ be the folding map. Let $e^{\prime}$ be an edge of $Y$, with endpoints $v_{0}^{\prime}, v_{1}^{\prime}$, such that $f\left(e^{\prime}\right)=q(e)$ and the fold occurs at $v_{0}^{\prime}$. Let $g$ be the generator of $\operatorname{Stab}_{G}(e)$ and $g^{m}$ the one of $\operatorname{Stab}_{G}\left(e^{\prime}\right)$. We know $m \neq 0$ since $G$ is freely indecomposable, and so $|m|>1$ since the fold is nontrivial ( $\operatorname{Stab}_{G}\left(e^{\prime}\right)$ is strictly contained in $\left.\operatorname{Stab}_{G}(e)=\operatorname{Stab}_{G}(q(e))\right)$. We may assume $m>1$, the case for $m<-1$ being analogous.

Define $Y_{0}, g^{k} Y_{1}$ for $k=0, \ldots, m-1$ to be the components of $Y$ minus the edges $g^{k} e^{\prime}$, containing $v_{0}^{\prime}, g^{k} v_{1}^{\prime}$ respectively. Also let $X_{0}, X_{1}$ be the components of $X-q(e)$ containing $q\left(v_{0}\right), q\left(v_{1}\right)$, and $T_{0}, T_{1}$ the ones of $T_{\Gamma}-e$ containing $v_{0}, v_{1}$. Observe that $f\left(Y_{0}\right)=X_{0}, f\left(g^{k} Y_{1}\right)=X_{1}, q\left(T_{0}\right)=X_{0}$ and $q\left(T_{1}\right)=X_{1}$.

Seeking a proof by contradiction, suppose $H$ is elliptic in $\Gamma_{1}$. Thus $H$ fixes a point $x^{\prime}$ in $Y$. Since $g \in H$ and $g$ fixes no point of $g^{k} Y_{1}$ for any $k$, we get $x^{\prime}$ must belong to $Y_{0}$. Then $H$ fixes the point $x=f\left(x^{\prime}\right)$ in $X_{0}$ and stabilizes the subtree $q^{-1}(x)$ in $T_{0}$.

Now, $e$ separates $q^{-1}(x)$ from $v_{1}$, and $H$ stabilizes both. So $H$ must also stabilize $e$, which is a contradiction, since $H$ contained $\operatorname{Stab}_{G}(e)$ strictly.

We can obtain Proposition 3.4 from this lemma as follows.

Proof of Proposition 3.4 Suppose that $\Gamma$ is a GBS graph in the conditions of Proposition 3.4. Notice that if $\Gamma$ is not a single vertex, then $G=\pi_{1}(\Gamma) \not \mathbb{Z}$ and so it is one-ended. If $\Gamma$ is not unfolded, then there is an edge $e$ of $\Gamma$ and a nontrivial unfolding $\Gamma_{1}$ of $\Gamma_{e}$. In the Bass-Serre tree $T_{\Gamma}$, let $v_{0}$ be the endpoint of $e$ at which the unfolding occurs, and $v_{1}$ be the other endpoint. Let $e^{\prime}$ be the edge of $T_{\Gamma_{1}}$ with stabilizer contained in $\operatorname{Stab}_{G}(e)$. Put $H=\operatorname{Stab}_{G}\left(v_{1}\right)$. Then $\operatorname{Stab}_{G}\left(e^{\prime}\right) \leq \operatorname{Stab}_{G}(e) \leq H$, where both inclusions are strict (the first one because the unfolding is nontrivial, the second one by the hypothesis of Proposition 3.4). These three subgroups are infinite cyclic, and $\operatorname{Stab}_{G}\left(e^{\prime}\right)$ is elliptic in $\Gamma_{1}$, so $H$ must also be elliptic in $\Gamma_{1}$ (if $g^{n}$ acts elliptically on a tree, so acts $g$ ). This contradicts Lemma 4.1.

The following is the main result of this section. It gives an unfoldedness criterion for universal $\mathbb{Z}$-splittings.

Theorem 4.2 Let $G$ be a one-ended group. Suppose $\Gamma$ is a reduced $\mathbb{Z}$-splitting of $G$ satisfying universality. If every edge group is a proper subgroup of its neighboring vertex groups, then it is unfolded, and is therefore a cyclic JSJ decomposition for $G$. 
Proof Again, suppose that $\Gamma$ is not unfolded. Let $e$ be an edge of $\Gamma$ and $\Gamma_{1}$ a nontrivial unfolding of $\Gamma_{e}$. Let $v_{0}$ and $v_{1}$ be the endpoints of $e$, when considered in $T_{\Gamma}$, and assume the unfolding occurs at $v_{0}$.

By the universality of $\Gamma$, it has a refinement $\widehat{\Gamma}$, obtained as in Theorem 2.14(4), that admits a morphism $T_{\widehat{\Gamma}} \rightarrow T_{\Gamma_{1}}$. Let $w_{0}, w_{1}$ be the vertices of $e$ as an edge of $T_{\widehat{\Gamma}}$, that correspond to $v_{0}, v_{1}$ respectively. Put $H=\operatorname{Stab}_{G}\left(w_{1}\right)$.

Since $H$ is elliptic in $\widehat{\Gamma}$ and there is a morphism $T_{\widehat{\Gamma}} \rightarrow T_{\Gamma_{1}}$, then $H$ must also be elliptic in $\Gamma_{1}$.

On the other hand, $H \leq \operatorname{Stab}_{G}\left(v_{1}\right)$ and it contains $\operatorname{Stab}_{G}(e)$. If $v_{1}$ is not a $\mathrm{QH}$ vertex, then it does not get split in the refinement $\widehat{\Gamma}$. So $H=\operatorname{Stab}_{G}\left(v_{1}\right)$, which contains $\operatorname{Stab}_{G}(e)$ strictly by hypothesis. If $v_{1}$ is a $\mathrm{QH}$ vertex with $G_{v_{1}}=\pi_{1}(S)$, then $H$ is conjugate to $\pi_{1}\left(S_{0}\right)$, where $S_{0}$ is a component of $S$ cut by some weakly essential simple closed curves. Thus $H$ is not cyclic, and so must contain $\operatorname{Stab}_{G}(e)$ strictly.

By the lemma, $H$ cannot be elliptic in $\Gamma_{1}$, which is a contradiction.

\section{Adding surface vertices to universal graphs}

In this section we deduce the universality of a $\mathbb{Z}$-splitting, given the universality of certain subgraphs of it. We start with some preliminaries.

Definition 5.1 Let $\Gamma$ be a $\mathbb{Z}$-splitting of a finitely generated group and $e$ an edge in $\Gamma$. Let $v^{+}$and $v^{-}$be the endpoints of $e$, and $a$ be a generator of $G_{e}$. Define $m_{e}^{+}$ as the supremum of the $m$ such that $\partial_{e}^{+}(a)=b^{m}$ for some $b \in G_{v^{+}}$. Define $m_{\boldsymbol{e}}^{-}$in the same manner.

The number $m_{e}^{+}$will be called the label of $e$ at the endpoint $v^{+}$. (With some abuse of notation, for when $e$ is a loop, it gets two labels, one for each boundary map). We remark that it is possible to have $m_{e}^{+}=+\infty$, although this will not happen in the cases that concern us. If $v^{+}$is a $\mathrm{QH}$ vertex with $G_{v^{+}}=\pi_{1}(S)$, then the element $b$ in the definition is the class of the boundary component of $S$ corresponding to $\partial_{e}^{+}$. In particular $m_{e}^{+}$is finite. Also, in the case when $G_{v}$ is cyclic, the element $b$ is a generator of $G_{v^{+}}$and the label is also finite.

The following theorem, due to Zieschang, will be crucial in the proof of Theorem 5.5. The proof is referred, and the corollary results from iterated use of the theorem. 
Theorem 5.2 [20, Theorem 4.12.1, page 140] Let $S$ be a 2-orbifold with boundary components $\gamma_{1}, \ldots, \gamma_{n}$. Let $\Delta$ be a one-edged $\mathbb{Z}$-splitting of $\pi_{1}(S)$ in which $\left[\gamma_{1}\right], \ldots,\left[\gamma_{n}\right]$ are elliptic. Then there is a weakly essential simple closed curve $c$ in $S$ such that $\Delta$ is obtained by cutting $S$ along $c$ (via Van-Kampen's theorem).

Corollary 5.3 Let $S$ be a 2 -orbifold with boundary components $\gamma_{1}, \ldots, \gamma_{n}$. If $\Delta$ is a general $\mathbb{Z}$-splitting in which $\left[\gamma_{1}\right], \ldots,\left[\gamma_{n}\right]$ are elliptic, then $\Delta$ is obtained by cutting $S$ along $c_{1}, \ldots, c_{m}$, disjoint weakly essential simple closed curves.

We also need the following simple lemma about coverings of surfaces and 2-orbifolds.

Lemma 5.4 Let $S$ be a connected 2-orbifold with boundary that is neither a disk nor a cylinder with cone-points. Then there is a 4-sheeted cover $\hat{S}$ of $S$, such that every boundary component $\gamma$ of $S$ is covered by two boundary components $\hat{\gamma}_{0}, \hat{\gamma}_{1}$ of $\widehat{S}$, and each one is a double cover of $\gamma$.

Proof Assume $S$ is an orientable surface, the general case is analogous. Write

$$
\pi_{1}(S)=\left\langle a_{1}, \ldots, a_{g}, b_{1}, \ldots, b_{g}, p_{1}, \ldots, p_{m} \mid \Pi_{k} p_{k} \Pi_{j}\left[a_{j}, b_{j}\right]=1\right\rangle .
$$

Observe if the genus is positive, then the kernel of the map $\pi_{1}(S) \rightarrow \mathbb{Z}_{2}$ sending $a_{1}$ to 1 and all other generators to 0 defines a double cover $S_{0}$ of $S$ where each boundary component of $S$ is covered by two homeomorphic copies of itself. On the other hand, when $m$ is even, the map $\pi_{1}(S) \rightarrow \mathbb{Z}_{2}$ sending all $p_{i}$ to 1 and $a_{j}, b_{j}$ to 0 is a well defined homomorphism, and its kernel gives a double cover $S_{1}$ of $S$ in which each boundary component of $S$ is covered twice by a single boundary curve of $S_{1}$. Notice $S_{1}$ always has positive genus, by the Euler characteristic computation for a finite cover.

Combining these covers produces the desired 4-sheeted cover in the cases when $m$ is even, or $m$ is odd but $S$ has positive genus. There remains the case of a sphere with an odd number of punctures. In this case, we have

$$
\pi_{1}(S)=\left\langle p_{1}, \ldots, p_{m} \mid p_{1} \cdots p_{m}=1\right\rangle .
$$

Consider the map $\pi_{1}(S) \rightarrow \mathbb{Z}_{2} \times \mathbb{Z}_{2}$ sending $p_{1}, \ldots, p_{m-2}$ to $(1,0), p_{m-1}$ to $(0,1)$ and $p_{m}$ to $(1,1)$. This map is a well defined homomorphism and gives the desired covering.

The following result is the main point of this section. Under some conditions, it allows us to recognize the universality of a $\mathbb{Z}$-splitting built from the union of smaller universal graphs and some extra $\mathrm{QH}$ vertices. 
Theorem 5.5 Let $\Gamma$ be a $\mathbb{Z}$-splitting of the one-ended group $G$. Let $V=\left\{v_{1}, \ldots, v_{m}\right\}$ be a subset of the $Q H$ vertices of $\Gamma$, such that their corresponding 2-orbifolds are not disks nor cylinders with cone-points. Let $\Gamma_{1}, \ldots, \Gamma_{k}$ be the components of the subgraph spanned by the vertices not in $V$, and put $G_{i}=\pi_{1}\left(\Gamma_{i}\right)$. Assume

(1) If $e$ is an edge with endpoints in $V$, then $m_{e}^{+}, m_{e}^{-}>1$.

(2) If the vertices $v_{j} \in V$ and $w \in \Gamma_{i}$ are connected by an edge, then $w$ is not a $Q H$ vertex of $\Gamma_{i}$.

(3) Each $G_{i}$ is one-ended, and each $\Gamma_{i}$ satisfies universality as a $\mathbb{Z}$-splitting of $G_{i}$.

Then $\Gamma$ satisfies universality.

Proof First we observe that if $w$ is a vertex of $\Gamma_{i}$, then it is $\mathrm{QH}$ in $\Gamma$ if and only if it is $\mathrm{QH}$ in $\Gamma_{i}$. If $w$ is $\mathrm{QH}$ in $\Gamma_{i}$, then it has no more incident edges in $\Gamma$ by (2), and so it is also $\mathrm{QH}$ in $\Gamma$. And if $w$ is $\mathrm{QH}$ in $\Gamma$, then it cannot be connected by an edge to $v_{j} \in V$, for that would cause $G_{i}$ to be freely decomposable. (To see that, let $p_{1}, \ldots, p_{n}$ be the boundary classes in $G_{w}$, and suppose that the edge assigned to $p_{1}$ is not in $\Gamma_{i}$. Observe that $p_{2}, \ldots, p_{n}$ are part of a free basis for $G_{w}$. This induces a free splitting of $G_{w}$ that allows us to refine $\Gamma_{i}$ to a graph with some trivial edge groups).

Let $\Gamma^{\prime}$ be a $\mathbb{Z}$-splitting of $G$, and $T^{\prime}=T_{\Gamma^{\prime}}$ its Bass-Serre tree. Consider the action of $G_{i}$ on $T^{\prime}$ by restriction of the action of $G$. Passing to a minimal invariant subtree, $G_{i}$ acts cocompactly and with cyclic edge stabilizers (since $G_{i}$ is one-ended). So this action gives rise to a $\mathbb{Z}$-splitting of $G_{i}$. By universality of $\Gamma_{i}$, there is a refinement $\widehat{\Gamma}_{i}$ and a morphism $T_{\widehat{\Gamma}_{i}} \rightarrow T^{\prime}$ so that $\widehat{\Gamma}_{i}$ is obtained from $\Gamma_{i}$ by splitting QH vertex groups along weakly essential simple closed curves. Then all the non-QH vertex groups, and all the edge groups of $\Gamma_{i}$ are elliptic in $\Gamma^{\prime}$.

This proves that all the non-QH vertex groups of $\Gamma$ are elliptic in $\Gamma^{\prime}$, since $V$ consists only of QH vertices.

It also implies if an edge $e$ has an endpoint in some $\Gamma_{i}$, then $G_{e}$ is elliptic in $\Gamma^{\prime}$ : If $e$ is contained in $\Gamma_{i}$ we have already shown it. If $e$ has endpoints $v_{j} \in V$ and $w \in \Gamma_{i}$, then $w$ is non-QH by (2), and so $G_{w}$ is elliptic in $\Gamma^{\prime}$. Since $G_{e} \subset G_{w}$, then $G_{e}$ must also be elliptic in $\Gamma^{\prime}$.

Claim All edge groups of $\Gamma$ are elliptic in $\Gamma^{\prime}$.

Proof of the claim If $e$ has an endpoint in some $\Gamma_{i}$, we have already proved it.

Now let $e$ be an edge with endpoints $v^{ \pm} \in V$ (which can be the same vertex). 
Let $\gamma^{ \pm}$be the boundary components of the orbifolds $S^{ \pm}$corresponding to $G_{v^{ \pm}}$, so that $\partial_{e}^{ \pm}: G_{e} \rightarrow\left\langle\left[\gamma^{ \pm}\right]\right\rangle$. Let $H_{e}=\left\langle\left[\gamma^{+}\right],\left[\gamma^{-}\right]\right\rangle \subset G$ be the subgroup generated by the classes of $\gamma^{ \pm}$. Note that $H_{e}$ is a GBS group.

If either $m_{e}^{+}>2$ or $m_{e}^{-}>2$, then the splitting of $H_{e}$ with edge $e$ satisfies the conditions in Theorem 3.3, that are direct consequences of those over $m_{e}^{ \pm}$. So it is a JSJ decomposition of $H_{e}$, and so $G_{e}$ is elliptic in $T^{\prime}$ (as we have done for the $\Gamma_{i}$ ).

If $m_{e}^{+}=m_{e}^{-}=2$, we proceed by contradiction. Suppose $G_{e}$ is hyperbolic in $\Gamma^{\prime}$, and let $c$ be the generator of $G_{e}$. Take an edge $e^{\prime}$ of $\Gamma^{\prime}$ that has a lift to $T^{\prime}$ lying on the axis of $c$. Then $\Gamma^{\prime \prime}=\Gamma_{e^{\prime}}^{\prime}$ is a one-edged $\mathbb{Z}$-splitting of $G$ in which $c$ is hyperbolic. Let $T^{\prime \prime}=T_{\Gamma^{\prime \prime}}$ be its Bass-Serre tree, and let $a$ be the generator of the edge group of $\Gamma^{\prime \prime}$.

On one hand, we consider the subgroup $H_{e}$. Note that $H_{e}=\pi_{1}(K)$ where $K$ is a Klein bottle. ( $K$ is obtained by gluing two Möbius bands by their boundaries. In this case $\gamma^{+}$and $\gamma^{-}$are the core circles of the Möbius bands, and $c$ is their common boundary circle). The action of $H_{e}$ on $T^{\prime \prime}$ by restriction gives rise to a $\mathbb{Z}$-splitting of $H_{e}$ (for $H_{e}$ is freely indecomposable). Note that $c$ is hyperbolic in it, since it is so in $T^{\prime \prime}$. So this $\mathbb{Z}$-splitting is nontrivial, and we can take $b \in H_{e}$ a generator of an edge group. Now observe that the edge groups of this decomposition of $H_{e}$ are all conjugate in $G$ into $\langle a\rangle$. This is so because the only elements that fix an edge of $T^{\prime \prime}$ are the conjugates of a power of $a$. So we obtain an element $b \in H_{e}, b \neq 1$, which is conjugate to a power of $a$.

On the other hand, we consider the subgroup $M$ constructed as follows.

Take the graph formed by the vertices in $V$ and the edges of $\Gamma$ with endpoints in $V$ and both labels equal to 2 . Let $\Delta$ be the component of this graph that contains $e$. For each vertex $v_{j} \in \Delta$ write $G_{v_{j}}=\pi_{1}\left(S_{j}\right)$, where $S_{j}$ is the orbifold that corresponds to $v_{j}$ as a $\mathrm{QH}$ vertex of $\Gamma$. Let $\widehat{S}_{j}$ be the 4 -sheeted cover of $S_{j}$ given by Lemma 5.4. These covers can be extended to a 4 -sheeted cover of the whole graph $\Delta$, that can be constructed as follows. Define the graph $\widehat{\Delta}$ to have the same vertices as $\Delta$, with $\pi_{1}\left(\hat{S}_{j}\right)<\pi_{1}\left(S_{j}\right)$ as vertex group at $v_{j}$. And for each edge $f$ of $\Delta$, we put in four edges $f_{0}, f_{1}, f_{2}$ and $f_{3}$ in $\widehat{\Delta}$, with infinite cyclic edge groups. The boundary maps are described as follows: Suppose $v_{j}$ is an endpoint of $f$ and $\delta$ is the boundary component of $S_{j}$ corresponding to $f$. Then let $\delta_{0}$ and $\delta_{1}$ be the boundary components of $\hat{S}_{j}$ that cover $\delta$ and assign $f_{0}, f_{2}$ to $\delta_{0}$ and $f_{1}, f_{3}$ to $\delta_{1}$. So the generator of $G_{f_{0}}$ maps to $\left[\delta_{0}\right]$ and similarly for the others. This is a 4 -sheeted cover, in the sense that $\pi_{1}(\widehat{\Delta})<\pi_{1}(\Delta)$ with index 4 . (This is best seen by building a presentation 2 -complex of $\pi_{1}(\Delta)$, using $S_{j}$ for the vertex $v_{j}$, and tubes for the edges. Then extend the covers 
$\widehat{S}_{j}$ of $S_{j}$ to covers of the tubes.) Note that the labels of the edges of $\widehat{\Delta}$ are all 1 . The local picture at each edge is as in the example on Figure 1.

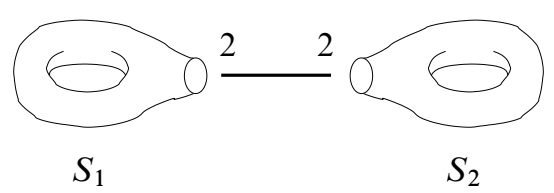

(a)

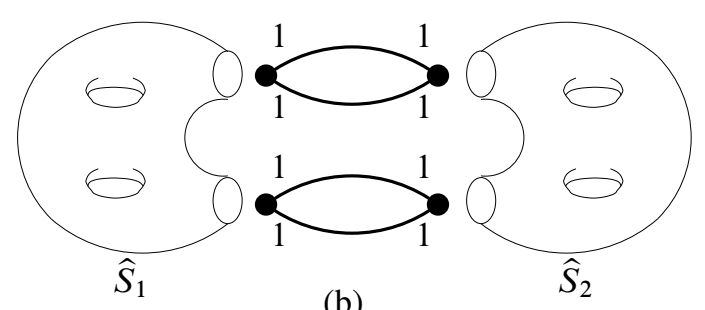

(b)

Figure 1: Example of the cover $\widehat{\Delta}$

(a) Original graph $\Delta$, with two QH vertices and an edge with $m_{e}^{ \pm}=2$

(b) Its cover $\widehat{\Delta}$

Now let $M$ be the subgroup of $\pi_{1}(\widehat{\Delta})$ generated only by the vertex groups and the stable letters of the $f_{0}$ and $f_{1}$ edges. This is equivalent to saying that $M$ is the fundamental group of the graph resulting from $\widehat{\Delta}$ by erasing all $f_{2}$ and $f_{3}$ edges (and keeping the $f_{0}$ and $f_{1}$ edges). So $M=\pi_{1}(S)$, where $S$ is the orbifold that results from gluing the $\hat{S}_{j}$ along their boundary curves, so that two boundary curves are identified if they are connected by an edge of $\widehat{\Delta}$. Note that in this subgroup, $c$ is the class of one of the common boundaries of $\widehat{S}^{+}$and $\widehat{S}^{-}$that corresponds to a lift of the edge $e$. (Say, to $e_{0}$ ). Lets call this curve $\beta$, so that $[\beta]=c$.

If $p$ is a boundary curve of $S$, then some power of $[p]$ is in an edge group $G_{f}$ of $\Gamma$, so that $f$ is not in $\Delta$. (All boundaries corresponding to edges in $\Delta$ were glued). Since $f$ is not in $\Delta$, but connects to a vertex in $\Delta$, we know that $f$ is one of the edges for which we have already proved that $G_{f}$ is elliptic in $\Gamma^{\prime}$. Thus, the classes of the boundary curves of $S$ are elliptic in $\Gamma^{\prime}$ (and so in $\Gamma^{\prime \prime}$ ).

Again, restrict to $M$ the action on $T^{\prime \prime}$. This gives a $\mathbb{Z}$-splitting of $M$, in which $c$ is hyperbolic and all the boundary classes of $S$ are elliptic. By Corollary 5.3, this decomposition of $M$ is obtained by cutting $S$ along disjoint, weakly essential simple closed curves. Let $\alpha$ be one of these curves, so that it intersects $\beta$ essentially (ie the intersection cannot be removed by homotopy). There must be such $\alpha$, since $c=[\beta]$ is hyperbolic in this decomposition.

Now, since $[\alpha]$ is a generator of an edge group in the $\mathbb{Z}$-splitting of $M$ induced by $T^{\prime \prime}$, then $[\alpha]$ must be conjugate in $G$ to a power of $a$. This is by the same argument we used for the element $b$.

Since both $[\alpha]$ and $b$ are conjugate to a power of $a$, then they have the same dynamics in every action of $G$ on a tree. That is to say, in a given $G$-tree, they are either both elliptic or both hyperbolic, depending on the behaviour of $a$. 
For the contradiction, consider $\Gamma^{*}$, the one-edged splitting of $G$ over $\left[\gamma^{-}\right]$. This splitting is obtained from $\Gamma_{e}$ by folding at $v^{+}$. In the case of an amalgamation, $\Gamma_{e}$ corresponds to $A *\langle c\rangle$ and $\Gamma^{*}$ to $A *\left\langle\left[\gamma^{-}\right]\right\rangle\left(H_{e} *\langle c\rangle, B\right)$. The case of an HNN extension is similar.

In both cases $H_{e}$ is contained in a vertex group, so $b$ must be elliptic in $\Gamma^{*}$. We will show $[\alpha]$ is hyperbolic in $\Gamma^{*}$. This will give the contradiction, thus proving the claim.

Consider the action of $M$ on $T_{\Gamma^{*}}$ by restriction. It gives a splitting of $M=\pi_{1}(S)$ in which the boundary classes are elliptic, so we may use Corollary 5.3 again. This time $c=[\beta]$ stabilizes an edge on $T_{\Gamma^{*}}$, thus $\beta$ is one of the curves that cut $S$ to form this decomposition. Since $\alpha$ intersects $\beta$ essentially, then $[\alpha]$ must be hyperbolic in this splitting of $M$, and therefore in $\Gamma^{*}$.

Thus far we know that all non-QH vertex groups and all edge groups of $\Gamma$ are elliptic in $\Gamma^{\prime}$. For each QH vertex $v$ of $\Gamma$, write $G_{v}=\pi_{1}\left(S_{v}\right)$ where $S_{v}$ is the corresponding orbifold. Then $G_{v}$ acts on $T^{\prime}$ by restriction. Since edge groups of $\Gamma$ are elliptic in $\Gamma^{\prime}$, it follows that the boundary classes of $S_{v}$ act elliptically on $T^{\prime}$. Applying Corollary 5.3, the $\mathbb{Z}$-splitting of $G_{v}$ induced by its action on $T^{\prime}$ is obtained by cutting $S_{v}$ along some disjoint, weakly essential simple closed curves. The vertex groups of this decomposition correspond to the pieces of $S_{v}$ after the cutting, and are elliptic in $\Gamma^{\prime}$. Also note that each boundary curve of $S_{v}$ lies in exactly one of these pieces. So the splitting of $G_{v}$ is compatible with $\Gamma$, giving rise to a refinement of $\Gamma$.

Let $\widehat{\Gamma}$ be the refinement of $\Gamma$ that results from splitting all the QH vertex groups $G_{v}$ as above. Then all vertex and edge groups of $\hat{\Gamma}$ are elliptic on $\Gamma^{\prime}$. Equivalently, there is a morphism $T_{\widehat{\Gamma}} \rightarrow T^{\prime}$. Since $\Gamma^{\prime}$ was an arbitrary $\mathbb{Z}$-splitting of $G$, this concludes the proof.

\section{Quadratic Baumslag-Solitar graphs}

Now we consider graphs of groups $\Gamma$ with edge groups infinite cyclic, and vertex groups either $\mathrm{QH}$ surface groups or infinite cyclic. We will call these graphs quadratic Baumslag-Solitar (QBS) graphs. For simplicity, we restrict the $\mathrm{QH}$ vertex groups to be surface groups instead of general 2-orbifold groups. Notice that in a GBS graph all labels are finite, and easily computed from the boundary maps as indicated in the remarks after Definition 5.1.

A group $G$ will be called a $Q B S$ group if it can be written as $\pi_{1}(\Gamma)$, where $\Gamma$ is a QBS graph. 
If $\Gamma$ is a QBS graph, let $\Gamma_{1}, \ldots, \Gamma_{k}$ be the components of the subgraph spanned by the non-QH vertices. That is, the components that are left after removing all $\mathrm{QH}$ vertices and the edges connecting to them. Note that each $\Gamma_{i}$ is then a GBS graph. The $\Gamma_{i}$ will be called the GBS components of $\Gamma$.

A GBS component of $\Gamma$ will be called a leaf if it is reduced to a single vertex $w$, and is attached to only one edge $e$ with $G_{w}=G_{e}$.

Proposition 6.1 Let $\Gamma$ be a reduced QBS graph with no leaves, and $G=\pi_{1}(\Gamma)$. Assume $G \not \mathbb{Z}$. Then $G$ is one ended.

This is a corollary of [19, Theorem 18].

We now turn to the main theorem of the paper. It allows us to recognize the defining graph of a QBS group as a Rips-Sela JSJ decomposition, in most cases.

Theorem 1.1 Let $\Gamma$ be a QBS graph, $G=\pi_{1}(\Gamma)$. Suppose that $\Gamma$ is reduced, has no leaves, and satisfies the following conditions:

(1) Each edge $e$ of $\Gamma$ has labels $m_{e}^{+}, m_{e}^{-}>1$.

(2) Each GBS component $\Gamma_{i}$ of $\Gamma$ is reduced, and $T_{\Gamma_{i}}$ is not a point or a line.

Then $\Gamma$ is a Rips-Sela JSJ decomposition for $G$.

Proof Let $V$ be the set of $\mathrm{QH}$ vertices of $\Gamma$. The components of $\Gamma$ minus $V$ are the GBS components $\Gamma_{i}$ of $\Gamma$. By condition (1) and Proposition 3.4, each $\Gamma_{i}$ is unfolded. This, together with condition (2), allows us to apply Theorem 3.3 (Forester's result). We conclude each $\Gamma_{i}$ is a JSJ decomposition of $G_{i}=\pi_{1}\left(\Gamma_{i}\right)$. By Lemma 3.2, the $G_{i}$ are one-ended. By these facts and condition (1), we have verified the hypotheses of Theorem 5.5 for $\Gamma$ and $V$. So $\Gamma$ satisfies universality. Now we can use Theorem 4.2 to conclude that $\Gamma$ is unfolded. Therefore $\Gamma$ is a JSJ decomposition of $G$, by Corollary 2.16 .

When some edge label equals 1 , then $\Gamma$ may fail to be a JSJ decomposition. This was already true for GBS graphs. In Figure 2 there is an example, in which the edge $e$ with a label equal to 1 is not in a GBS component. However, if in the same figure we change the label 1 for some $m_{e}^{-}>1$, and make $k=1$ instead, we do get a JSJ decomposition (by Theorem 5.5 and then Theorem 4.2), which is not covered by Theorem 1.1. 


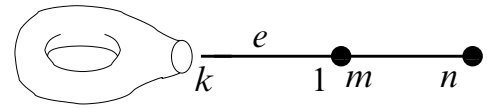

(a)

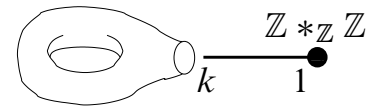

(b)

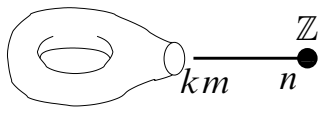

(c)

Figure 2: (a) A QBS graph that satisfies universality (for $m, n>1$ ), but with $m_{e}^{-}=1$. It admits an unfolding at the surface vertex, as shown in (b) and (c).

(b) The one-edged splitting corresponding to the edge $e$ of the graph in (a)

(c) An unfolding of the splitting in (b)

\section{References}

[1] M Bestvina, M Feighn, Bounding the complexity of simplicial group actions on trees, Invent. Math. 103 (1991) 449-469 MR1091614

[2] B H Bowditch, Cut points and canonical splittings of hyperbolic groups, Acta Math. 180 (1998) 145-186 MR1638764

[3] M J Dunwoody, M E Sageev, JSJ-splittings for finitely presented groups over slender groups, Invent. Math. 135 (1999) 25-44 MR1664694

[4] M J Dunwoody, E L Swenson, The algebraic torus theorem, Invent. Math. 140 (2000) 605-637 MR1760752

[5] M Forester, Deformation and rigidity of simplicial group actions on trees, Geom. Topol. 6 (2002) 219-267 MR1914569

[6] M Forester, On uniqueness of JSJ decompositions of finitely generated groups, Comment. Math. Helv. 78 (2003) 740-751 MR2016693

[7] K Fujiwara, P Papasoglu, JSJ-decompositions of finitely presented groups and complexes of groups, Geom. Funct. Anal. 16 (2006) 70-125 MR2221253

[8] V Guirardel, G Levitt, JSJ decompositions: definitions, existence and uniqueness I: The JSJ deformation space arXiv:0911.3173

[9] V Guirardel, G Levitt, JSJ decompositions: definitions, existence and uniqueness II: Compatibility and acylindricity arXiv:1002.4564

[10] V Guirardel, G Levitt, A general construction of JSJ decompositions, from: "Geometric group theory", (GN Arzhantseva, L Bartholdi, J Burillo, E Ventura, editors), Trends Math., Birkhäuser, Basel (2007) 65-73 MR2395790

[11] W H Jaco, P B Shalen, Seifert fibered spaces in 3-manifolds, Mem. Amer. Math. Soc. 21 (1979) viii+192 MR539411

[12] K Johannson, Homotopy equivalences of 3-manifolds with boundaries, Lecture Notes in Mathematics 761, Springer, Berlin (1979) MR551744

[13] P H Kropholler, An analogue of the torus decomposition theorem for certain Poincaré duality groups, Proc. London Math. Soc. 60 (1990) 503-529 MR1044309 
[14] E Rips, Z Sela, Cyclic splittings of finitely presented groups and the canonical JSJ decomposition, Ann. of Math. 146 (1997) 53-109 MR1469317

[15] P Scott, G A Swarup, Regular neighbourhoods and canonical decompositions for groups, Astérisque 289, Soc. Math. France (2003) MR2032389

[16] Z Sela, Structure and rigidity in (Gromov) hyperbolic groups and discrete groups in rank 1 Lie groups. II, Geom. Funct. Anal. 7 (1997) 561-593 MR1466338

[17] J-P Serre, Trees, Springer, Berlin (1980) MR607504 Translated from the French by John Stillwell

[18] J Stallings, Group theory and 3-dimensional manifolds, Yale Math. Monogr. 4, Yale Univ. Press, New Haven, CT (1971) MR0415622 James K Whittemore Lecture in Math. (Yale Univ.)

[19] H Wilton, One-ended subgroups of graphs of free groups with cyclic edge groups, Geom. Topol. 16 (2012) 665-683

[20] H Zieschang, E Vogt, H-D Coldewey, Surfaces and planar discontinuous groups, Lecture Notes in Mathematics 835, Springer, Berlin (1980) MR606743 Translated from the German by John Stillwell

Department of Mathematics, Cornell University

310 Malott Hall, Ithaca, NY 14853, USA

alonso@math. cornell.edu

http://www. math. cornell. edu/People/PhD/alonso.html

Received: 7 November $2011 \quad$ Revised: 20 June 2012 